\title{
Device for handling electron microscopy grids
}

\author{
Mika Lännenpää and Juhani E. Syväoja \\ University of Joensuu, Joensuu, Finland
}

BioTechniques 40:450-452 (April 2006)

doi 10.2144/000112147

The handling of grids and transferring them individually between drops on a sheet of Parafilm with forceps is often cumbersome. Inserting grids into small slits of a piece of silicone tubing for staining has been described previously (1). Here we present a novel device for the easy handling of grids attached to a piece of silicon tubing, allowing both chemical staining and immunoreactions.

The base of the device is made of a Teflon ${ }^{\circledR}$ bar by lathe. The upper part of the base is fitted into the neck of a $34 \times 24 \mathrm{~mm}$ glass bottle, and the broad top serves as a cap (Figure 1A). The lower slim part has a narrowing of $3 \times$ $8.5 \mathrm{~mm}$ at the bottom, where the piece of silicon tubing is fitted. An L-shaped hole with a diameter of $3 \mathrm{~mm}$ is drilled through the slim part to allow for the free escape of air from the tubing when it is submerged into staining solutions. A haft screw is fitted into threads of the cap. A 10-mm-long bit is cut from $12-\mathrm{mm}$ silicon tubing. Slits of $1 \mathrm{~mm}$ are cut into the edge with a scalpel. A broader mark slit indicates the order of the slits.

The grids are inserted into the slits using forceps. They can be identified according to their order compared with the mark slit. About $1.5 \mathrm{~mL}$ staining solution is enough to cover the grids hanging near the bottom of the bottle (Figure 1B). The grids are easily transferred simultaneously from one bottle to another for staining and rinsing. The excess solution can be removed using a piece of filter paper. The silicon tubing holds the grids quite tightly, and it is possible to rinse them rather vigorously by turning the device backward and forward (or lifting up and down) in the bottle.

For immunoreactions, the blocking solution is removed, and a drop of antibody dilution is pipeted onto each grid. A volume of $2.5-5 \mu \mathrm{L}$ is adequate to cover a grid that is considerably less than the volume needed for conventional drop incubation methods (Figure $1 C)$. The device is placed into a $50 \times 24$ $\mathrm{mm}$ bottle where the grids hang above a small volume of water or phosphatebuffered saline (PBS). This forms a humidified incubation chamber that prevents the evaporation of the drops.

The device is ideal for handling up to 10 grids, especially for immunological reactions and subsequent contrasting. We have used nickel mesh grids, but all types of grids could be used. The handling of grids is far more convenient and faster than incubations in drops on a sheet of Parafilm. The soft silicon tubing is also an ideal material for holding grids, because Formvar coating and sections are often damaged due to the extensive use of forceps during the traditional drop incubations. Furthermore, a minimum volume of antibody dilution is required. The device is easy to transfer into appropriate incubation temperatures. Also, the incubation times can be followed more precisely. The system also suits well to microwave antigen retrieval procedures, provided a beaker is used instead of a bottle.

The commercial grid staining devices are designed for either simultaneous staining of grids, without the possibility to treat them individually, or only the individual treatment of each grid. Our device combines the advantages of these systems with a minimum consumption of solutions and is easier and faster to use.

The correct handling of the device and loading the drops is easy to learn after practicing a few times using water. It is important to keep the silicon tubing clean and carefully remove all traces of the blocking solution with a piece of filter paper or Kimwipes ${ }^{\circledR}$
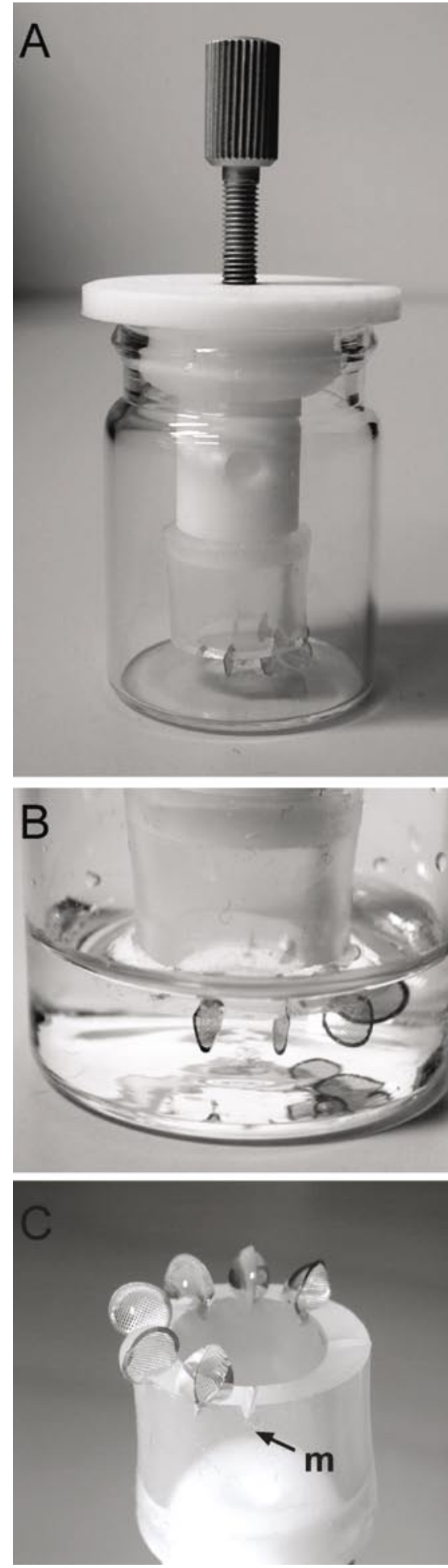

Figure 1. Electron microscopy grid holder. (A) The device in an incubation bottle. (B) Grids in a staining solution. The mirror images of the grids are seen on the bottom of the bottle. (C) Drops of antibody dilution on grids. $\mathrm{m}$, the mark slit according to which the order of grids may be easily determined. 


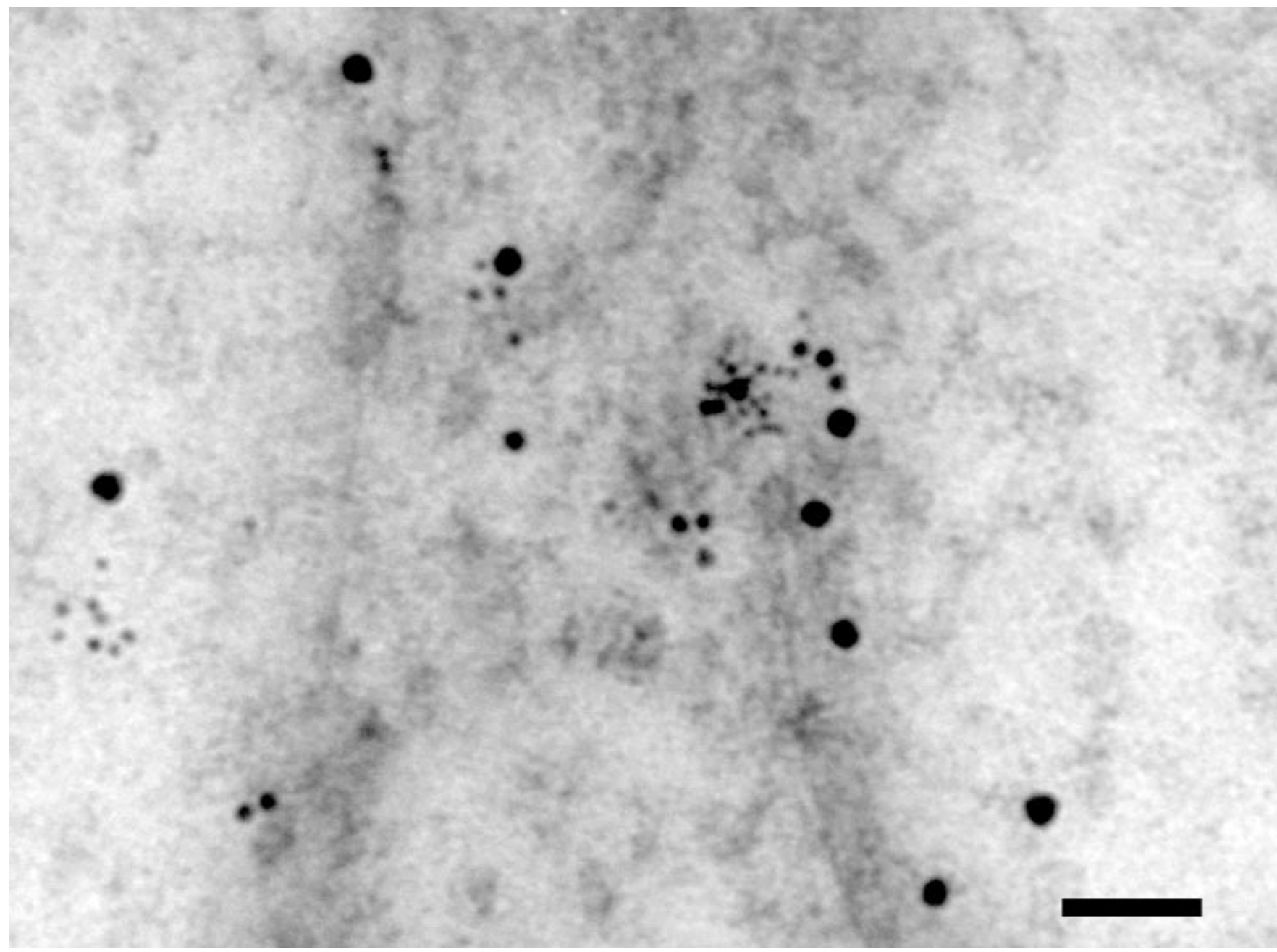

Figure 2. Immunolabeling obtained using the electron microscopy grid holder. This triple immunolabeling demonstrates the colocalization of bromodeoxyuridine $(6 \mathrm{~nm})$, DNA polymerase $\varepsilon(10 \mathrm{~nm})$, and DNA ligase $1(15 \mathrm{~nm})$ in a HeLa cell nucleus. Bromodeoxyuridine labeling corresponds to newly replicated DNA. Scale bar, $50 \mathrm{~nm}$.

before applying the antibody drops. It is also crucial not to apply too much antibody dilution to prevent the mixing of the drops. Although the drops are prevented from moving, even if the device is placed upside down (as shown in Figure 1C), we recommend that solutions be pipeted onto the grids when they are hanging down. It should be noted that a small area of the edge of the grid is inside the slit at all times and is not exposed to the incubation solutions. However, it is usually easy to position the grids so that the sections are well apart from the slits.

The device is routinely used in our laboratory and has replaced the other methods. The results are comparable to those obtained with the traditional staining and incubation system (Figure 2). We have used mainly the immunolabeling procedure described by Jaunin et al. (2), however, the system could be applied to any drop incubation procedure.

\section{COMPETING INTERESTS STATEMENT}

The authors declare no competing interests.

\section{REFERENCES}

1. Dykstra, M.J. and L.E. Reuss. 2003. Biological Electron Microscopy. Theory, Techniques, and Troubleshooting, p. 205. Kluwer Academic/Plenum Publishers, NY.
2. Jaunin, F., A.E. Visser, D. Cmarko, J.A. Aten, and S. Fakan. 2000. Fine structural in situ analysis of nascent DNA movement following DNA replication. Exp. Cell Res. 260:313-323.

Received 16 January 2006; accepted 30 January 2006.

Address correspondence to Mika Lännenpä̈̈, Department of Biology, University of Joensuu, P.O. Box 111, FIN-80101 Joensuu, Finland. e-mail: mika.lannenpaa@joensuu.fi

To purchase reprints

of this article, contact

Reprints@BioTechniques.com 